\title{
The Impact Of LIFO In The Fortune 500 In 2007
}

John Kostolansky, Loyola University Chicago, USA

\begin{abstract}
Recent legislative consideration to end the use of the Last-in, First-out (LIFO) inventory method, as well as the movement to adopt international accounting standards which do not permit LIFO, have created anew the debate over how important LIFO is to U.S. businesses. This paper catalogs the use of LIFO during 2007 among the largest 500 U.S. companies by analyzing disclosures from the Form 10-K annual report (or the corporate annual report for privately-held firms). Analysis of the data provides evidence of the frequency of use of LIFO, the financial impact on reported income and on reported assets due to its use, and the particular industry categories that are the major beneficiaries of the method.
\end{abstract}

Keywords: Last-in First-out, LIFO, inventory analysis, IFRS, SEC Roadmap

\section{INTRODUCTION}

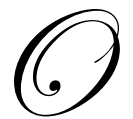

ver the last half century, the Last-in, First-out (LIFO) accounting method has been highlighted in the media, researched by academics, evaluated by stock analysts, and debated in Congress. In 1957 Time Magazine observed that "To many a U.S. corporation, LIFO is a magic formula in times of inflation. It cuts their profits for tax purposes without taking a penny out of their coffers" (Time Magazine, February 1957). Serious debate erupted in 2006, when Congress proposed to eliminate the use of LIFO for tax reporting.

Testimony before the Senate Committee on Finance by Professor George Plesko of the University of Connecticut affirmed that the use of LIFO has declined steadily since the early 1980's from approximately 70 percent of large firms to about 40 percent in 2004. Plesko gave further evidence that large firms from one-third of all industry categories and most small publicly-traded firms do not use LIFO. Finally, Plesko noted that most businesses are privately held, and while there is little public data for these firms, there is evidence that most use the first-in, first-out (FIFO) accounting method.

A contrary position on LIFO was presented to the Committee by The LIFO Coalition, an industry group formed in response to the LIFO-elimination proposal. The Coalition argued that "Professor Plesko's testimony significantly understates the use of LIFO by the U.S. business community and the very substantial adverse effect of repeal on the U.S. economy, with such inaccuracies based in part on inclusion of irrelevant data and failure to recognize accounting protocols that create differences between statements of book and tax LIFO reserves.

In 2007, the Securities and Exchange Commission (SEC) approved the use of International Financial Reporting Standards (IFRS) by foreign private issuing companies and considered allowing or requiring U.S. firms to also use IFRS. Since IFRS does not allow LIFO, the issue raised by the Congressional tax proposals resurfaced and debate over its impact resumed. The August 2008 SEC release containing a proposed roadmap for the potential mandatory adoption of International Financial Reporting Standards (IFRS) by issuers in the US in 2014 brings the focus on LIFO full to a peak.

This paper catalogs the use of LIFO in 2007 among the largest 500 U.S. companies. To mitigate the potential industry bias inherent in a single index, both the Fortune 500 and Standard \&Poor's (S\&P) 500 companies were examined. Using disclosures in the Management Discussion and Analysis and footnote sections of the Form $10-\mathrm{K}$ annual report (or the corporate annual report for privately-held firms), information about the company's 
inventory and its valuation methods was gathered for 2007 and 2006. The data are analyzed to determine the relative popularity of LIFO, its general impact on earnings, and industry preference.

\section{KEY FINDINGS}

Databases of inventory information were constructed for firms comprising the 2007 Fortune 500 and the 2007 Standard and Poor's 500 using footnote disclosures in Form 10-K or the company's annual report. Each database includes the amount of inventory, the inventory method (where applicable), the size of LIFO Reserve, and the effect of any LIFO liquidations on income. Analysis of the data shed light on the frequency with which LIFO was used, as well as the frequency of use of the other methods, and the proportion of firms which carry no inventory.

Data analysis also provided interesting insight into how reported profits would have increased if an alternate inventory method had been used instead of the LIFO method. Since oil companies, whose profits have been recently criticized, were major benefactors from using LIFO, the impact of LIFO on profits takes on increased significance. Also of interest is the greatly increased inventory value that would have been reported in the absence of LIFO.

Several key findings of this study are:

1. In the Fortune 500, 135 companies reported using LIFO for valuing all or some of their inventory. Given that 141 Fortune 500 firms do not carry inventory, the proportion of firms choosing LIFO was 38 percent (135/359). The S\&P 500 results were similar.

2. LIFO is employed in $\mathbf{3 7}$ of the $\mathbf{5 4}$ Fortune $\mathbf{5 0 0}$ industry categories whose firms carry inventory. Another 18 industry categories, such as banking and insurance, do not report inventory.

3. Exxon Mobil topped the list of firms with the largest LIFO Reserves, reporting a reserve of $\$ 25.4$ billion. The next 9 largest LIFO Reserve amounts ranged between $\$ 1.4$ billion and $\$ 7.0$ billion.

4. Fewer than 20 LIFO firms in the Fortune 500 would have seen their 2007 net income increase by more than 2 percent if their inventory had been valued on a more current basis. However, there are several firms whose net income would have increased significantly. Sunoco's 2007 net income of $\$ 891$ million would have more than doubled to an estimated $\$ 1.9$ billion if LIFO had not been used as the inventory method. Exxon's net income would have increased about 15 percent, or $\$ 6.2$ billion.

5. The total LIFO Reserves of the 135 LIFO companies in the Fortune 500 grew to $\$ 82$ billion, up 39 percent from the 2006 level of $\$ 59$ billion. Most of this increase occurred in the oil sector. Note that the value of the LIFO Reserves for tax reporting purposes cannot be calculated from information in the $10-\mathrm{K}$ but is thought to be higher.

6. Thirty six LIFO firms would have reported at least a 20 percent increase in inventory under an alternative inventory method. At the top of this list, Sunoco's inventory would have quadrupled from $\$ 1.1$ billion to over $\$ 5$ billion if LIFO had not been used. Exxon Mobil's inventory would have tripled from $\$ 11.1$ billion to $\$ 36.5$ billion.

7. Not surprisingly, the LIFO Reserve of major oil and energy firms increased sharply in 2007 as the price of oil surged to all-time highs. Ten such firms recorded an increase of more than 50 percent in their LIFO Reserves. Marathon Oil had the largest relative increase of 140 percent, jumping from $\$ 1.7$ billion to $\$ 4.0$ billion.

\section{USE OF LIFO}

As was mentioned in the Introduction, the extent of use of the LIFO method has been debated in recent years. This study documents the frequency of use of various inventory methods across the industry categories that comprise the Fortune 500 firms. Table 1 details the 72 industry categories that comprise the Fortune $500 .{ }^{1}$

\footnotetext{
${ }^{1}$ There were 74 individual groups that were collapsed to 72 for this study. The industry group "insurance: life, health was identified in two separate categories, one for mutual companies and one for stock companies. The same was true for the industry group "insurance: property \& casualty.
} 
Table 1

Number of Fortune 500 Firms by Industry Category

\begin{tabular}{|c|c|}
\hline Industry Category & Total \\
\hline Advertising, marketing & 2 \\
\hline Aerospace \& defense & 10 \\
\hline Airlines & 7 \\
\hline Apparel & 4 \\
\hline Automotive retailing, services & 8 \\
\hline Beverages & 6 \\
\hline Building materials, glass & 2 \\
\hline Chemicals & 17 \\
\hline Commercial banks & 21 \\
\hline Computer peripherals & 3 \\
\hline Computer software & 2 \\
\hline Computers, office equipment & 8 \\
\hline Diversified financials & 9 \\
\hline Diversified outsourcing & 1 \\
\hline Electronics, electrical equipment & 4 \\
\hline Energy & 13 \\
\hline Engineering, construction & 5 \\
\hline Entertainment & 6 \\
\hline Financial data services & 4 \\
\hline Food \& drug stores & 10 \\
\hline Food consumer products & 14 \\
\hline Food production & 5 \\
\hline Food services & 4 \\
\hline Forest \& paper products & 3 \\
\hline Furniture & 1 \\
\hline General merchandisers & 10 \\
\hline Health care: insurance \& managed care & 7 \\
\hline Health care: medical facilities & 6 \\
\hline Health care: pharmacy \& other services & 5 \\
\hline Home equipment, furnishings & 3 \\
\hline Homebuilders & 11 \\
\hline Hotels, casinos, resorts & 5 \\
\hline Household \& personal products & 6 \\
\hline Industrial \& farm equipment & 13 \\
\hline Information technology services & 5 \\
\hline Insurance: life, health & 18 \\
\hline Insurance: property \& casualty & 20 \\
\hline Internet services \& retailing & 6 \\
\hline Mail, package, freight delivery & 2 \\
\hline Medical products \& equipment & 5 \\
\hline Metals & 8 \\
\hline Mining, crude oil production & 9 \\
\hline Miscellaneous & 3 \\
\hline Motor vehicle \& parts & 15 \\
\hline Network \& other communications equipment & 6 \\
\hline Oil \& gas equipment, services & 5 \\
\hline Packaging, containers & 7 \\
\hline Payroll services & 1 \\
\hline Petroleum refining & 10 \\
\hline Pharmaceuticals & 9 \\
\hline Pipelines & 6 \\
\hline Publishing, printing & 4 \\
\hline Railroads & 4 \\
\hline Real estate & 2 \\
\hline Savings institutions & 2 \\
\hline Scientific, photo, control equipment & 3 \\
\hline Securities & 7 \\
\hline Semiconductors \& other electronic components & 7 \\
\hline Specialty retailers & 24 \\
\hline Telecommunications & 13 \\
\hline
\end{tabular}


Table 1 continued

Number of Fortune 500 Firms by Industry Category

\begin{tabular}{|l|c|}
\hline \multicolumn{1}{|c|}{ Industry Category } & Total \\
\hline Temporary help & 2 \\
Tobacco & 2 \\
Toys, sporting goods & 1 \\
Transportation \& logistics & 2 \\
Transportation equipment & 2 \\
Trucking, truck leasing & 2 \\
Utilities: gas \& electric & 26 \\
Waste management & 2 \\
Wholesalers: diversified & 9 \\
Wholesalers: electronics \& office equipment & 7 \\
Wholesalers: food \& grocery & 4 \\
Wholesalers: health care & 5 \\
\hline Grand Total & 500 \\
\hline
\end{tabular}

Table 2

Number of Fortune 500 Firms Using LIFO, by Industry Category

\begin{tabular}{|c|c|}
\hline Fortune 500 Industry Category & Number \\
\hline Chemicals & 13 \\
\hline Industrial \& farm equipment & 11 \\
\hline Food \& drug stores & 9 \\
\hline Petroleum refining & 9 \\
\hline General merchandisers & 7 \\
\hline Motor vehicle \& parts & 7 \\
\hline Metals & 6 \\
\hline Specialty retailers & 5 \\
\hline Utilities: gas \& electric & 5 \\
\hline Wholesalers: diversified & 5 \\
\hline Aerospace \& defense & 4 \\
\hline Energy & 4 \\
\hline Food consumer products & 4 \\
\hline Household \& personal products & 4 \\
\hline Packaging, containers & 4 \\
\hline Wholesalers: health care & 4 \\
\hline Pharmaceuticals & 3 \\
\hline Beverages & 2 \\
\hline Building materials, glass & 2 \\
\hline Electronics, electrical equipment & 2 \\
\hline Food production & 2 \\
\hline Forest \& paper products & 2 \\
\hline Home equipment, furnishings & 2 \\
\hline Insurance: property \& casualty & 2 \\
\hline Publishing, printing & 2 \\
\hline Tobacco & 2 \\
\hline Transportation equipment & 2 \\
\hline Wholesalers: food \& grocery & 2 \\
\hline Apparel & 1 \\
\hline Computers, office equipment & 1 \\
\hline Diversified financials & 1 \\
\hline Furniture & 1 \\
\hline Medical products \& equipment & 1 \\
\hline Mining, Crude oil products & 1 \\
\hline Oil \& gas equipment, services & 1 \\
\hline Scientific, photo, control equipment & 1 \\
\hline Wholesalers: electronics \& office equipment & 1 \\
\hline Grand Total & 135 \\
\hline
\end{tabular}


The use of the LIFO inventory method is widespread, spanning 37 of the 72 industry groups comprising the Fortune 500 and including 135 of the 359 Fortune firms that carry inventory. Table 2 identifies the industry groups where LIFO is employed, listed in declining order of frequency of use.

When examining the use of a particular inventory method within an industry group, it must be remembered that a single company may simultaneously use more than one inventory method and that a conglomerate firm will be grouped in its main industry category. Thus, not only will the number of inventory methods sum to over 500 but also there may be firms reporting inventory in industry categories where inventory is not expected. Table 3 reports the number of firms by industry which use LIFO exclusively or in combination with other inventory methods.

Table 3

Number of Fortune 500 Firms Using LIFO And Other Inventory Methods, By Industry Category ( $\mathrm{L}=\mathrm{LIFO}, \mathrm{AC}=$ average cost, $\mathrm{F}=\mathrm{FIFO}, \mathrm{SP}=$ specific identification)

\begin{tabular}{|c|c|c|c|c|c|c|}
\hline Industry Category & $\mathbf{L}$ & $\mathrm{L}, \mathbf{A C}$ & $\mathbf{L}, \mathbf{F}$ & $\mathbf{L}, \mathbf{F}, \mathbf{A C}$ & $\mathrm{L}, \mathrm{AC}, \mathrm{F}, \mathrm{SP}$ & Total \\
\hline Aerospace \& defense & & 1 & 2 & 1 & & 4 \\
\hline Apparel & & & 1 & & & 1 \\
\hline Beverages & 1 & & 1 & & & 2 \\
\hline Building materials, glass & & & 1 & 1 & & 2 \\
\hline Chemicals & 2 & 2 & 7 & 2 & & 13 \\
\hline Computers, office equipment & & & 1 & & & 1 \\
\hline Diversified financials & & & 1 & & & 1 \\
\hline Electronics, electrical equipment & & & 1 & 1 & & 2 \\
\hline Energy & & 4 & & & & 4 \\
\hline Food \& drug stores & 7 & & 2 & & & 9 \\
\hline Food consumer products & & & 3 & 1 & & 4 \\
\hline Food production & & & 2 & & & 2 \\
\hline Forest \& paper products & & 1 & & 1 & & 2 \\
\hline Furniture & & & 1 & & & 1 \\
\hline General merchandisers & 5 & & 2 & & & 7 \\
\hline Home equipment, furnishings & & & 2 & & & 2 \\
\hline Household \& personal products & & & 2 & 2 & & 4 \\
\hline Industrial \& farm equipment & 4 & & 7 & & & 11 \\
\hline Insurance: property \& casualty & 1 & & & & 1 & 2 \\
\hline Medical products \& equipment & & & 1 & & & 1 \\
\hline Metals & 1 & 1 & 3 & 1 & & 6 \\
\hline Mining, crude oil products & & 1 & & & & 1 \\
\hline Motor vehicle \& parts & & & 6 & 1 & & 7 \\
\hline Oil \& gas equipment, services & & 1 & & & & 1 \\
\hline Packaging, containers & & 2 & 1 & 1 & & 4 \\
\hline Petroleum refining & 3 & 3 & 1 & 2 & & 9 \\
\hline Pharmaceuticals & 2 & & 1 & & & 3 \\
\hline Publishing, printing & 1 & & 1 & & & 2 \\
\hline Scientific, photo, control equipment & 1 & & & & & 1 \\
\hline Specialty retailers & 2 & & 3 & & & 5 \\
\hline Tobacco & 1 & & 1 & & & 2 \\
\hline Transportation equipment & & & 2 & & & 2 \\
\hline Utilities: gas \& electric & & 5 & & & & 5 \\
\hline Wholesalers: diversified & 2 & 1 & 2 & & & 5 \\
\hline Wholesalers: electronics \& office equipment & & & $\frac{2}{1}$ & & & 1 \\
\hline Wholesalers: food \& grocery & & & 2 & & & 2 \\
\hline Wholesalers: health care & 1 & & 3 & & & 4 \\
\hline Grand Total & 34 & 22 & 64 & 14 & 1 & 135 \\
\hline
\end{tabular}

\section{LIFO Reserves}

Since the LIFO method calculates cost of goods sold using the "last-in" (newer) prices, a company's inventory will be valued using older and typically lower prices. As a consequence, LIFO inventories will typically 
have a market value considerably greater than their accounting value. This price differential is referred to as the LIFO Reserve. The LIFO Reserve is the cumulative differential between LIFO pricing for inventory and an alternative inventory valuation method. As such, the Reserve represents the cumulative income differential that a firm would have reported over the time period it has been using LIFO.

Which industries garner the most benefit from using LIFO can be partially answered by looking at the size of the LIFO Reserves across each industry. Table 4 reports the total dollars of LIFO Reserve for each of the Fortune 500 industries where LIFO was used. The total value of the LIFO Reserve across all 500 firms was $\$ 82,371$ million. Two-thirds of that resided in the petroleum refining industry, with the remainder scattered across 36 other industries. The apparel and specialty retailer groups reported using LIFO but had no LIFO Reserve.

Table 4

2007 LIFO Reserves in the Fortune 500 By Industry Category (Dollar amounts in millions)

\begin{tabular}{|c|c|}
\hline $\begin{array}{l}\text { Industry Category } \\
\end{array}$ & LIFO Reserves \\
\hline Petroleum refining & $\$ 56,267$ \\
\hline Industrial \& farm equipment & 4,693 \\
\hline Metals & 3,716 \\
\hline Chemicals & 3,668 \\
\hline Motor vehicle \& parts & 2,828 \\
\hline Food \& drug stores & 2,786 \\
\hline Wholesalers: diversified & 1,007 \\
\hline Utilities: gas \& electric & 934 \\
\hline Tobacco & 751 \\
\hline Aerospace \& defense & 729 \\
\hline Diversified financials & 623 \\
\hline Insurance: property \& casualty & 482 \\
\hline Forest \& paper products & 459 \\
\hline Wholesalers: food \& grocery & 445 \\
\hline Energy & 329 \\
\hline Packaging, containers & 325 \\
\hline Wholesalers: health care & 313 \\
\hline Household \& personal products & 280 \\
\hline Food production & 235 \\
\hline Beverages & 232 \\
\hline Electronics, electrical equipment & 175 \\
\hline Food consumer products & 165 \\
\hline Transportation equipment & 148 \\
\hline Pharmaceuticals & 135 \\
\hline Oil \& gas equipment, services & 116 \\
\hline Mining, crude oil products & 102 \\
\hline Building materials, glass & 90 \\
\hline Publishing, printing & 78 \\
\hline Furniture & 64 \\
\hline Home equipment, furnishings & 62 \\
\hline Wholesalers: electronics \& office equipment & 60 \\
\hline General merchandisers & 29 \\
\hline Computers, office equipment & 24 \\
\hline Scientific, photo, control equipment & 18 \\
\hline Medical products \& equipment & 4 \\
\hline Apparel & 0 \\
\hline Specialty retailers & 0 \\
\hline Total LIFO Reserves 2007 & $\$ 82,371$ \\
\hline
\end{tabular}

The relative importance of LIFO to individual firms can be seen in Table 5 which reports the 10 largest LIFO Reserves for 2007. 
Table 5

The Ten Largest LIFO Reserves for 2007: Fortune 500

(Dollar amounts in millions)

\begin{tabular}{|l|c|c|}
\hline \multicolumn{1}{|c|}{ Company } & Industry Category & LIFO Reserve 2007 \\
\hline Exxon Mobil & Petroleum refining & \$25,400 \\
\hline Chevron & Petroleum refining & 658 \\
\hline ConocoPhillips & Petroleum refining & 6,2068 \\
\hline Valero Energy & Petroleum refining & 4,034 \\
\hline Marathon Oil & Petroleum refining & 3,868 \\
\hline Sunoco & Petroleum refining & 2,617 \\
\hline Caterpillar & Industrial \& farm equipment & 1,511 \\
\hline Dow Chemical & Chemicals & 1,423 \\
\hline General Motors & Motor vehicle \& parts & 1,400 \\
\hline Tesoro & Petroleum refining & \\
\hline
\end{tabular}

As expected, the firms experiencing the most significant difference in inventory valuation caused by LIFO are in the petroleum refining industry. Interestingly, only one of the 10 firms in this category, Frontier Oil, did not use LIFO to value any part of its inventory.

As for which firms would have experienced the largest relative balance sheet impact, Table 6 identifies those firms whose inventory value would have increased by over 50 percent if LIFO had not been used. Petroleum refiners are again prominent on the list. Sunoco's inventory would have more than quadrupled using an alternative inventory method. Exxon Mobil's inventory would have tripled, while six other petroleum refiners would have reported more than double the LIFO value of inventory.

Table 6

Fortune 500 Companies With Adjusted Value for Inventory Greater Than 150\% of LIFO Value (\$ millions) (Dollar amounts in millions)

\begin{tabular}{|c|c|c|c|c|c|}
\hline Company & Industry Category & $\begin{array}{c}\text { (Adjusted } \\
\text { Value of } \\
\text { Inventory) } \\
\div \\
\text { (Book Value) }\end{array}$ & $\begin{array}{c}2007 \\
\text { Inventory } \\
+ \text { LIFO } \\
\text { Reserve }\end{array}$ & $\begin{array}{c}\text { LIFO } \\
\text { Reserve } \\
2007\end{array}$ & $\begin{array}{c}\text { Inventory } \\
2007\end{array}$ \\
\hline Sunoco & Petroleum refining & $436.35 \%$ & $\$ 5,018$ & $\$ 3,868$ & $\$ 1,150$ \\
\hline Exxon Mobil & Petroleum refining & $329.06 \%$ & 36,489 & 25,400 & 11,089 \\
\hline ConocoPhillips & Petroleum refining & $257.90 \%$ & 10,891 & 6,668 & 4,223 \\
\hline Valero Energy & Petroleum refining & $248.18 \%$ & 10,384 & 6,200 & 4,184 \\
\hline Chevron & Petroleum refining & $231.04 \%$ & 12,268 & 6,958 & 5,310 \\
\hline Marathon Oil & Petroleum refining & $223.10 \%$ & 7,311 & 4,034 & 3,277 \\
\hline Tesoro & Petroleum refining & $216.67 \%$ & 2,600 & 1,400 & 1,200 \\
\hline Murphy Oil & Petroleum refining & $213.42 \%$ & 1336 & 710 & 626 \\
\hline NiSource & Utilities: gas \& electric & $205.02 \%$ & 939 & 481 & 458 \\
\hline Eastman Chemical & Chemicals & $194.62 \%$ & 1049 & 510 & 539 \\
\hline AK Steel Holding & Metals & $183.31 \%$ & 1186 & 539 & 647 \\
\hline Hess & Petroleum refining & $182.32 \%$ & 2,279 & 1,029 & 1,250 \\
\hline Loews & Insurance: property \& casualty & $167.41 \%$ & 375 & 151 & 224 \\
\hline Deere & Industrial \& farm equipment & $152.76 \%$ & 3,570 & 1,233 & 2,337 \\
\hline
\end{tabular}

While the size of the LIFO Reserve indicates the cumulative difference in inventory value, the change in the LIFO Reserve from year to year indicates the income differential resulting from using the LIFO method. It is again interesting to note which industries reported the largest changes in their LIFO Reserve in 2007. Table 7 identifies the change in LIFO Reserve across the industry for each of the industries where LIFO was used. 
Table 7

Change in Value of 2007 LIFO Reserves By Industry Category for the Fortune 500 Firms

\begin{tabular}{|c|c|}
\hline Industry Category & Change in LIFO Reserve (in \$millions) \\
\hline Petroleum refining & $\$ 21,489$ \\
\hline Chemicals & 529 \\
\hline Industrial \& farm equipment & 395 \\
\hline Energy & 318 \\
\hline Metals & 236 \\
\hline Food production & 212 \\
\hline Food \& drug stores & 194 \\
\hline Utilities: gas \& electric & 108 \\
\hline Wholesalers: diversified & 99 \\
\hline Aerospace \& defense & 65 \\
\hline Insurance: property \& casualty & 63 \\
\hline Diversified financials & 59 \\
\hline Beverages & 50 \\
\hline Packaging, containers & 35 \\
\hline Mining, crude oil products & 28 \\
\hline Food consumer products & 24 \\
\hline Wholesalers: food \& grocery & 23 \\
\hline Oil \& gas equipment, services & 22 \\
\hline Pharmaceuticals & 22 \\
\hline Household \& personal products & 21 \\
\hline Motor vehicle \& parts & 18 \\
\hline Electronics, electrical equipment & 14 \\
\hline Transportation equipment & 11 \\
\hline Wholesalers: electronics \& office equipment & 8 \\
\hline Scientific, photo, control equipment & 7 \\
\hline Computers, office equipment & 3 \\
\hline Home equipment, furnishings & 2 \\
\hline Building materials, glass & 1 \\
\hline Medical products \& equipment & 0 \\
\hline Apparel & 0 \\
\hline Specialty retailers & 0 \\
\hline Furniture & (7) \\
\hline Publishing, printing & (7) \\
\hline Tobacco & (12) \\
\hline General merchandisers & (12) \\
\hline Forest \& paper products & (26) \\
\hline Wholesalers: health care & (54) \\
\hline Total Changes in LIFO Reserves 2007 & $\$ 23,938$ \\
\hline
\end{tabular}

Petroleum refining tops the list by a wide margin, indicating that profits in that industry would have been higher using other inventory methods. The dollar values of change taper quickly as one moves down the list.

Which individual firms would have felt the largest impact on earnings? Table 8 identifies the firms whose LIFO Reserve increased by over 50 percent in 2007.

Although several firms outside of the petroleum refining industry experienced large percentage increases in their LIFO Reserve, these increases were often based on relatively small Reserves. When large dollar amounts are considered, petroleum refining firms dominate this group, perhaps due to the large increases in the price of oil in 2007.

Viewing this factor from a different perspective, Table 9 shows the list of firms whose LIFO Reserve increased by more than $\$ 100$ million in 2007. This list is again dominated by firms in the petroleum refining industry, with several other industries bringing up the bottom of the list. 
Table 8

Fortune 500 Companies With LIFO Reserve Increases Over 50\% in 2007

(Dollar amounts in millions)

\begin{tabular}{|c|c|c|c|c|}
\hline \multicolumn{5}{|c|}{ mounts in mi } \\
\hline Company & Industry Category & $\begin{array}{c}\text { (2007 LIFO } \\
\text { Reserve) } \\
\div \\
\text { (2006 LIFO } \\
\text { Reserve) }\end{array}$ & $\begin{array}{l}\text { LIFO Reserve } \\
2007\end{array}$ & $\begin{array}{c}\text { LIFO Reserve } \\
2006\end{array}$ \\
\hline Integrys Energy Group & Energy & $\mathrm{n} / \mathrm{a}$ & $\$ 304$ & $\$ 0$ \\
\hline Archer Daniels Midland & Food production & 23.89 & 215 & 9 \\
\hline American Electric Power & Energy & 2.750 & 11 & 4 \\
\hline Marathon Oil & Petroleum refining & 2.40 & 4,034 & 1,682 \\
\hline Valero Energy & Petroleum refining & 2.14 & 6,200 & 2,900 \\
\hline Ryerson & Petroleum refining & 1.85 & 504 & 273 \\
\hline Murphy Oil & Petroleum refining & 1.83 & 710 & 389 \\
\hline Tesoro & Petroleum refining & 1.82 & 1,400 & 770 \\
\hline Sunoco & Petroleum refining & 1.70 & 3,868 & 2,273 \\
\hline Danaher & Scientific, photo, control equipment & 1.64 & 18 & 11 \\
\hline Exxon Mobil & Petroleum refining & 1.60 & 25,400 & 15,900 \\
\hline ConocoPhillips & Petroleum refining & 1.60 & 6,668 & 4,178 \\
\hline Hess & Petroleum refining & 1.52 & 1,029 & 676 \\
\hline Whole Foods Market & Food \& drug stores & 1.52 & 20 & 13 \\
\hline Nucor & Metals & 1.50 & 582 & 387 \\
\hline Black \& Decker & Industrial \& farm equipment & 1.50 & 15 & 10 \\
\hline
\end{tabular}

Table 9

Fortune 500 Companies: Increase in LIFO Reserve 2007 > \$100 Million (Dollar amounts in millions)

\begin{tabular}{|c|c|c|c|c|}
\hline Company & Industry Category & $\begin{array}{c}\text { Increase in } \\
\text { LIFO } \\
\text { Reserve }\end{array}$ & $\begin{array}{c}\text { LIFO Reserve } \\
\mathbf{2 0 0 7}\end{array}$ & $\begin{array}{c}\text { LIFO Reserve } \\
2006\end{array}$ \\
\hline Exxon Mobil & Petroleum refining & $\$ 9,500$ & $\$ 25,400$ & $\$ 15,900$ \\
\hline Valero Energy & Petroleum refining & 3,300 & 6,200 & 2,900 \\
\hline ConocoPhillips & Petroleum refining & 2,490 & 6,668 & 4,178 \\
\hline Marathon Oil & Petroleum refining & 2,352 & 4,034 & 1,682 \\
\hline Sunoco & Petroleum refining & 1,595 & 3,868 & 2,273 \\
\hline Chevron & Petroleum refining & 948 & 6,958 & 6,010 \\
\hline Tesoro & Petroleum refining & 630 & 1,400 & 770 \\
\hline Dow Chemical & Chemicals & 419 & 1,511 & 1,092 \\
\hline Hess & Petroleum refining & 353 & 1,029 & 676 \\
\hline Murphy Oil & Petroleum refining & 321 & 710 & 389 \\
\hline Integrys Energy Group & Energy & 304 & 304 & 0 \\
\hline Caterpillar & Industrial \& farm equipment & 214 & 2,617 & 2,403 \\
\hline Archer Daniels Midland & Food production & 206 & 215 & 9 \\
\hline Nucor & Metals & 195 & 582 & 387 \\
\hline Kroger & Food \& drug stores & 154 & 604 & 450 \\
\hline NiSource & Utilities: gas \& electric & 118 & 481 & 363 \\
\hline
\end{tabular}

As mentioned earlier, it is the change in the LIFO Reserve that determines the income differential that LIFO firms have experienced. The change in Reserve is a pretax number, so the after-tax income difference can be estimated at 65 percent of the change in LIFO Reserve (assuming a 35 percent tax rate). Rather than focus on the absolute dollar-value of change, which has already been specified in Table 9, the proportionate change in net income resulting from the use of an alternative inventory method is estimated. Table 10 is the list of Fortune 500 firms whose net income would have increased by over 10 percent if LIFO had not been used. 
Table 10

Fortune 500 Companies Adjusted Net Income More Than 10\% Greater Than LIFO Net Income (Dollar amounts in millions)

\begin{tabular}{|l|l|c|c|c|c|}
\hline & Industry Category & $\begin{array}{c}\text { (Adjusted Net Income) } \\
\mathbf{4} \\
\text { Company }\end{array}$ & $\begin{array}{c}\text { Adjusted Net } \\
\text { Income } \\
\mathbf{2 0 0 7}\end{array}$ & $\begin{array}{c}\text { LIFO Net } \\
\text { Income } \\
\mathbf{2 0 0 7}\end{array}$ & $\begin{array}{c}\text { 2007 Increase } \\
\text { in LIFO } \\
\text { Reserve }\end{array}$ \\
\hline Sunoco & Petroleum refining & 2.16 & $\$ 1,928$ & $\$ 891$ & 1,595 \\
\hline Integrys Energy Group & Energy & 1.78 & 452 & 254 & 304 \\
\hline Tesoro & Petroleum refining & 1.72 & 976 & 566 & 630 \\
\hline Valero Energy & Petroleum refining & 1.41 & 7,379 & 5,234 & 3,300 \\
\hline Marathon Oil & Petroleum refining & 1.39 & 5,485 & 3,956 & 2,352 \\
\hline Murphy Oil & Petroleum refining & 1.27 & 976 & 767 & 321 \\
\hline NiSource & Utilities: gas \& electric & 1.24 & 398 & 321 & 118 \\
\hline Exxon Mobil & Petroleum refining & 1.15 & 46,785 & 40,610 & 9,500 \\
\hline ConocoPhillips & Petroleum refining & 1.14 & 13,510 & 11,891 & 2,490 \\
\hline Hess & Petroleum refining & 1.13 & 2,061 & 1,832 & 353 \\
\hline Eastman Chemical & Chemicals & 1.10 & 330 & 300 & 46 \\
\hline
\end{tabular}

As can be seen, Sunoco's net income would have more than doubled from $\$ 891$ million to $\$ 1,928$ million if LIFO had not been used. Again, petroleum refiners would have reported substantially increased net income under an alternative inventory method. Given the interest by Congress in "excess oil profits," the adjusted profits estimated in Table 10 would have heightened Congressional interest.

\section{SUMMARY AND CONCLUSION}

While it may be true that the use of LIFO has declined in recent years, it is undeniable that LIFO is still widely used across the Fortune 500 by 38 percent of firms reporting inventory. The total LIFO Reserve across the Fortune 500 was $\$ 82,371$ million, an amount equal to 24 percent of the book value of the inventories of Fortune companies using LIFO and 12 percent of the book value of all inventories for the 500 companies.

LIFO causes significant differences in the reported value of inventory and net income for a small group of the Fortune 500 firms, many of which reside in the petroleum refining industry. At the extreme, inventory under LIFO is valued for one firm at 25 percent of a more current value and income for 2007 at less than half of what an alternative inventory method would report. Such extreme differences were limited to the top three to five firms being assessed. Although the exact tax consequences cannot be determined using the book accounting values, it can be estimated that $\$ 82,371$ million of income is deferred from taxation, delaying the payment of approximately 35 percent tax on that amount, or $\$ 28.8$ billion. More than 65 percent of this amount was in the petroleum refining industry.

\section{AUTHOR INFORMATION}

John Kostolansky is Associate Professor of Accounting at Loyola University Chicago. He was Chair of Loyola's Accounting Department (1979 - 1992) and Associate Dean for the School of Business Administration (1993 2005). He has since rejoined the faculty, teaching in the School's MBA, MSA, and EMBA Programs.

\section{REFERENCES}

1. Forms 10-K 2007 and Annual Reports 2007, Fortune 500 companies

2. Time Magazine, "LIFO v. FIFO," Monday, February 18, 1957, at www.time.com/time/magazine/article/0,9171,809159,00.html

3. United States Senate, Testimony of G.A. Plesko, University Of Connecticut School Of Business, before the Committee on Finance, June 13, 2006, p. $4-6$ at http://aaahq.org/ata/public-interest/notablecontributions/testimony/Plesko-Testimony-Enron.pdf

4. United States Senate Committee on Finance, LIFO Coalition Response to Plesko Testimony, p. 1, at www.sourcecorptax.com/senate/LIFO_Coalition_Response to_Plesko_Testimony.pdf 